\title{
Texture Directionality-Based Digital Watermarking in Nonsubsample Shearlet Domain
}

\author{
Jian Zhao, ${ }^{1}$ Shuaishuai Fan, ${ }^{1}$ Jian Jia, ${ }^{1,2}$ Shunli Zhang, ${ }^{1}$ Bo Jiang, \\ Wensheng $\mathrm{Xu},{ }^{1}$ and Wanru Zhang ${ }^{1}$ \\ ${ }^{1}$ School of Information Science and Technology, Northwest University, Xian 710127, China \\ ${ }^{2}$ School of Mathematics, Northwest University, Xian 710127, China \\ Correspondence should be addressed to Jian Jia; jiajian@nwu.edu.cn
}

Received 23 February 2017; Revised 23 April 2017; Accepted 10 May 2017; Published 12 June 2017

Academic Editor: Alessandro Lo Schiavo

Copyright (c) 2017 Jian Zhao et al. This is an open access article distributed under the Creative Commons Attribution License, which permits unrestricted use, distribution, and reproduction in any medium, provided the original work is properly cited.

Digital watermarking is a technique used to protect an author's copyright and has become widespread due to the rapid development of multimedia technologies. In this paper, a novel watermarking algorithm using the nonsubsample shearlet transform is proposed, which combines the directional edge features of an image. A shearlet provides an optimal multiresolution and multidirectional representation of an image based on distributed discontinuities such as edges, which ensures that the embedded watermark does not blur the image. In the proposed algorithm, the nonsubsample shearlet transform is used to decompose the cover image into directional subbands, where different directional subbands represent different directional and textured features. The subband whose texture directionality is strongest is selected to carry the watermark and is thus suitable for the human visual system. Next, singular value decomposition is performed on the selected subband image. Finally, the watermark is embedded in the singular value matrix, which is beneficial for the watermarking robustness and invisibility. In comparison with related watermarking algorithms based on discrete wavelet transforms and nonsubsample contourlet transform domains, experimental results demonstrate that the proposed scheme is highly robust against scaling, cropping, and compression.

\section{Introduction}

Given the rapid development of digital data and associated technologies, copyright protection of multimedia has become a challenging problem. In order to protect an author's ownership, the method of digital watermarking (a digital data hiding technique) provides an effective solution. A good digital watermarking algorithm must ensure that (1) the embedded watermark is visually imperceptible, (2) the reconstructed watermarked image has good visual quality, and (3) the watermark is resilient to attacks. In other words, a watermarking technique should resist watermark degradation due to common signal processing and geometric attacks.

A watermarking algorithm is regarded optimal if the scheme can balance the quality index of watermark robustness, imperceptibility, and capacity as proposed by Fridrich [1]. Watermarking can be performed in either the spatial domain (e.g., least significant bit) [2] or the transform domain (e.g., discrete Fourier transform (DFT) or discrete cosine transform (DCT)) [3, 4]. Moreover, the discrete wavelet transform (DWT) $[5,6]$ has the ability to capture 1D signal characteristics at different scales or resolutions, which makes the method widely applicable for image processing applications such as denoising and compression. However, DWT is unable to effectively represent multidimensional signals, and its limited directional filtering restricts watermarking capacity.

In order to overcome the limitations of the wavelet transform, several multiscale geometric analysis (MGA) tools such as ridgelet, directionlet, and contourlet transforms have been developed and successfully applied in image processing applications [7-12]. Shearlet, a multiresolution method, provides a tremendously effective representation of images containing edges $[13,14]$ and is widely used in watermarking algorithms [15-18]. Spread spectrum image watermarking in the discrete shearlet domain [15] employs the multiresolution 
representation characteristics of the DST to facilitate texture modeling for a given image. Zhao et al. [16] proposed a watermarking scheme based on the extended shearlet, where the watermark was embedded into the subband with the largest information entropy. Based on previous studies [17], a watermarking algorithm was proposed using the shearlet transform and bidiagonal singular value to achieve balance between robustness and imperceptibility.

In this paper, a nonsubsample shearlet transform (NSST), along with a combination of Laplace and shear filters, is used to decompose an image into different scales and directional subbands. The watermark can then be embedded into a special directional subband that achieves the best result. The proposed watermarking scheme has high robustness and imperceptibility depending on texture directionality in the NSST domain. The texture of an image with strong directionality reflects the contours and directional edges of the image, and the directional filter bank (DFB) of the NSST can effectively capture high-frequency content. The selection of a subband with strong texture directionality plays a significant role in the embedding and extraction steps of the watermarking algorithm.

The rest of this paper is organized as follows. Basic shearlet theory and related data are presented in Section 2. The proposed novel watermarking embedding and detection scheme is presented in Section 3. Experimental results are provided and discussed in Section 4, while important findings and conclusions are summarized in Section 5.

\section{Correlation with Basic Theory}

2.1. Shearlet Transform. Shearlets, or composite wavelets, provide an effective way to achieve a sparse directional image representation based on affine transformations and a combination of geometry and multiple scales. For dimension $n=2$, the affine system with composite dilations is described by

$$
\begin{aligned}
& \Lambda_{A B}\left\{\psi_{a, s, t}(x)=|\operatorname{det} A|^{a / 2} \psi\left(B^{s} A^{a} x-t\right): a, s \in R, t\right. \\
& \left.\quad \in R^{2}\right\},
\end{aligned}
$$

where $\psi \in L^{2}\left(R^{2}\right)$, A and $\mathbf{B}$ are $2 \times 2$ invertible matrices, and $|\operatorname{det} B|=1$. When $\mathbf{A}$ is the set $A_{a}=(a, 0 ; 0, \sqrt{a})(a>0)$, it is related to the scale transform, and the shear matrices $B_{s}=(1, s ; 0,1)$ are related to the area-preserving geometrical transform. The transform is described as [14]

$$
\begin{aligned}
\mathrm{SH}(\psi) & =\left\{\begin{array}{l}
\psi_{a, s, t}(x)=a^{-3 / 4} \psi\left(A_{a}^{-1} B_{s}^{-1} x-t\right) \\
=a^{-3 / 4} \psi\left(\left(\begin{array}{cc}
\frac{1}{a} & -\frac{s}{a} \\
0 & \frac{1}{\sqrt{a}}
\end{array}\right) x-t\right), a>0, s
\end{array}\right), a r
\end{aligned}
$$

$$
\left.\in\left[-\left(1+a^{1 / 2}\right), 1+a^{1 / 2}\right], t \in R^{2}\right\}
$$

We select $\psi$ such that

$$
\widehat{\psi}(\xi)=\widehat{\psi}\left(\xi_{1}, \xi_{2}\right)=\widehat{\psi}_{1}\left(\xi_{1}\right) \widehat{\psi}_{2}\left(\frac{\xi_{2}}{\xi_{1}}\right),
$$

where $\widehat{\psi}_{1}$ is a continuous wavelet for which $\widehat{\psi}_{1} \in C^{\infty}(R)$ with $\widehat{\psi}_{1} \subset[-2,1 / 2] \cup[1 / 2,2]$ and $\widehat{\psi}_{2}$ is selected to satisfy $\widehat{\psi}_{2} \in C^{\infty}(R)$ with $\widehat{\psi}_{2} \subset[-1,1], \widehat{\psi}_{2}>0$ on $(-1,1)$, and $\left\|\widehat{\psi}_{2}\right\|=1$. Under this assumption, the associated continuous shearlet transform of $f \in L^{2}\left(R^{2}\right)$ is defined as

$$
\mathrm{SH}_{f}(a, s, t)=\left\langle f, \psi_{a, s, t}\right\rangle \text {. }
$$

Sampling $\mathrm{SH}(\psi)$ on the discrete set of $a, s$, and $t$ is done as follows $[13,16]$ :

$$
\begin{aligned}
& a_{l}=2^{l} \quad(l \in Z), \\
& s_{j}=j * a_{l}^{1 / 2}=j * 2^{j / 2} \quad(k \in Z) .
\end{aligned}
$$

The associated discrete shearlet transform is described by

$$
\begin{gathered}
\left\{\psi_{j, l, k}^{(d)}(x)=\left|\operatorname{det} A_{d}\right|^{j / 2} \psi\left(B_{d}^{l} A_{d}^{j} x-k\right): j \geq 0,\right. \\
\left.-\left[2^{j / 2}\right] \leq l \leq\left[2^{j / 2}\right], k \in Z^{2}, d=0,1\right\} .
\end{gathered}
$$

2.2. Nonsubsample Shearlet Transform (NSST). The nonsubsample shearlet transform (NSST) is composed of the nonsubsample Laplacian pyramid transform and several different combinations of shearing filters. Figure 1(a) shows the flowchart for a nonsubsample shearlet where the Laplacian pyramid (LP) is used to capture point discontinuities and the directional filter bank (DFB) is used to link point discontinuities into linear structures. The DFB is designed to capture high-frequency content such as directional edges. An example of a frequency partition is shown in Figure 1(b).

A test image (zoneplate) is decomposed by NSST as shown in Figure 2. For LP decomposition, an approximate image is generated, and after DFB, 8 and 16 different directional subbands are obtained in the respective scale.

As observed in Figure 2, image directionality is strongly apparent after the shearlet transform. Different directional subbands represent the directional information related to different image details. Finally, the subband with the maximum texture directionality is selected for watermark embedding.

2.3. Singular Value Decomposition (SVD). Singular value decomposition (SVD) is a numerical statistical analysis tool that is used to effectively deal with matrices. Assume that an 


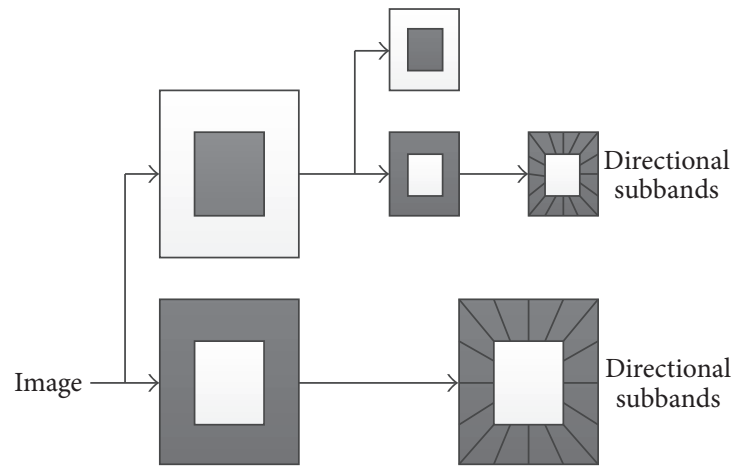

(a)

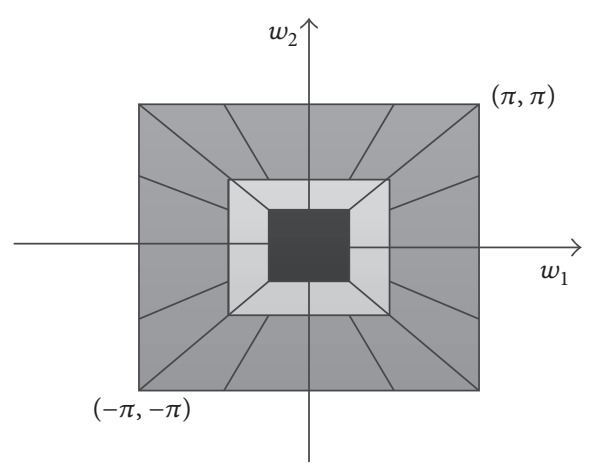

(b)

FIGURE 1: Diagram of shearlet transform: (a) illustration of succession of Laplacian and directional filtering and (b) example of frequencies by the shearlet transform.

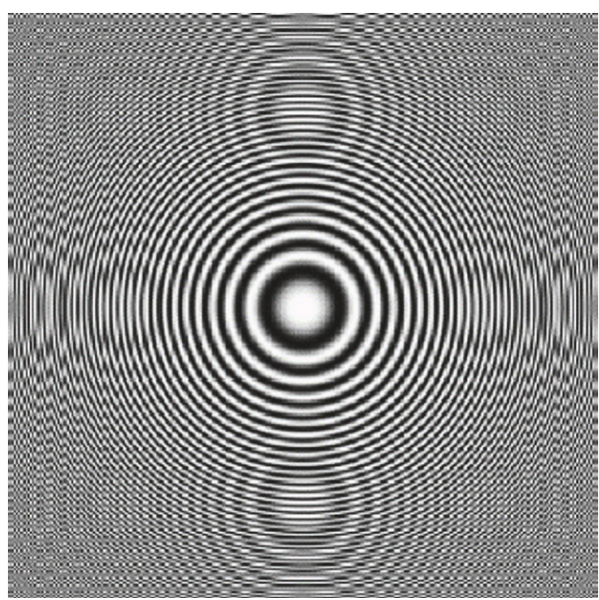

(a)
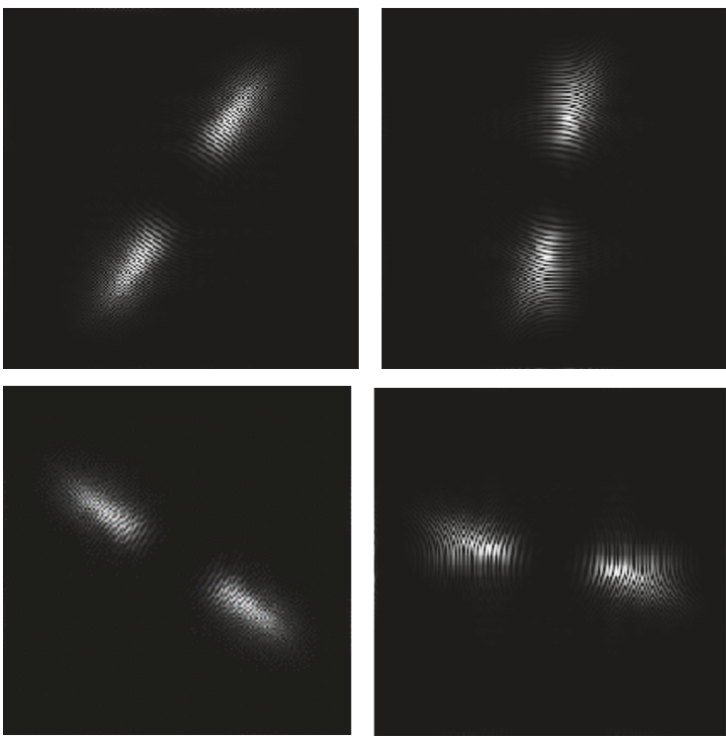

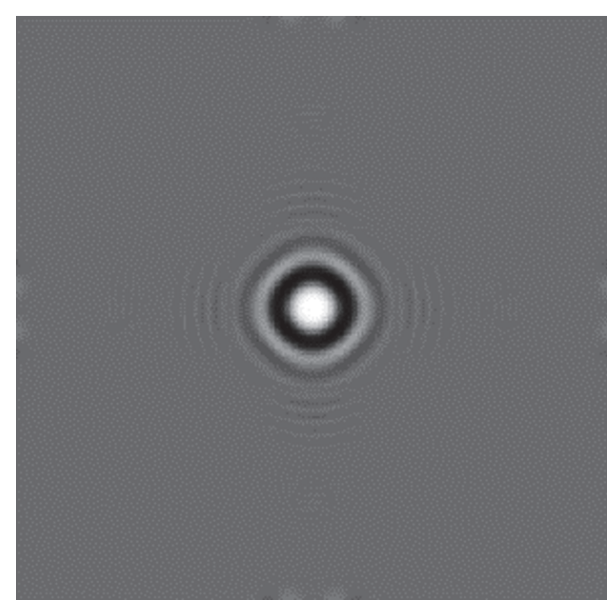

(b)
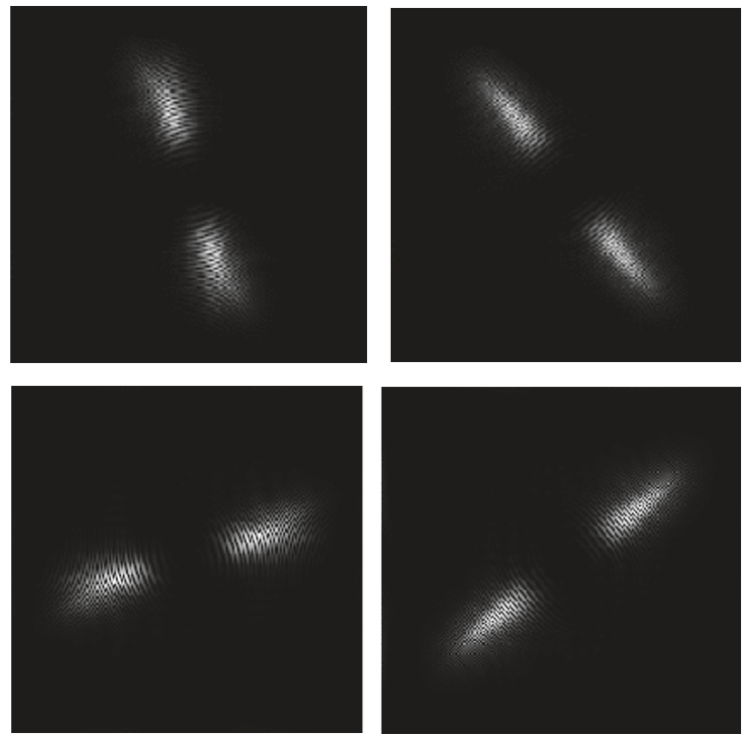

(c)

FIGURE 2: Shearlet decomposition of zoneplate image: (a) zoneplate, (b) approximate NSST coefficients, and (c) detailed coefficients. 
TABLE 1: Values of texture directionality.

\begin{tabular}{lcccccccc}
\hline Image & D1 & D2 & D3 & D4 & D5 & D6 & D7 & D8 \\
\hline Directionality & 0.3135 & 0.2673 & 0.2103 & 0.3321 & 0.1964 & 0.3111 & 0.3247 & 0.3007 \\
\hline
\end{tabular}

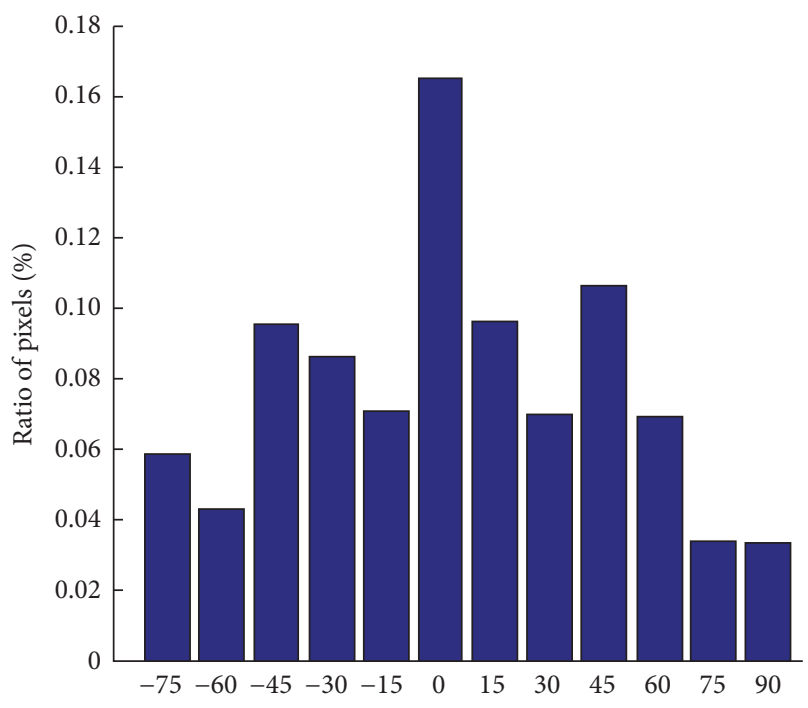

(a)

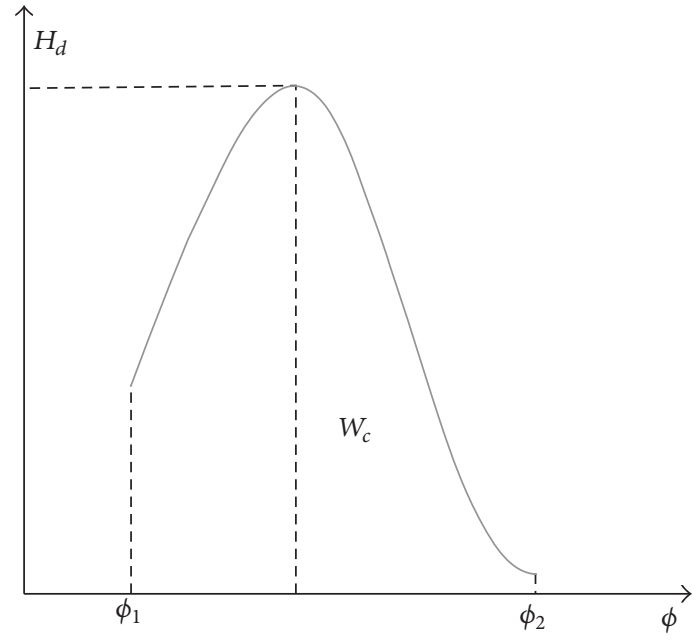

(b)

FIgURE 3: Directionality of texture: (a) direction edge histogram of Lena and (b) a peak in the edge histogram.

image is a nonnegative matrix. The singular value decomposition of matrix AA is defined as

$$
\begin{aligned}
\mathbf{A} \mathbf{A} & =U S V^{T} \\
& =\left[u_{1}, u_{2}, \ldots, u_{n}\right]\left[\begin{array}{llll}
\sigma_{1} & & & \\
& \sigma_{2} & & \\
& & \ddots & \\
& & & \sigma_{n}
\end{array}\right]\left[v_{1}, v_{2}, \ldots, v_{n}\right]^{T} \\
& =\sum_{i=1}^{n} \sigma_{i} u_{i} v_{i},
\end{aligned}
$$

where $U \in R^{m * n}$ and $V \in R^{m * n}$ are orthogonal matrices, $S \in$ $R^{m * n}$ is a diagonal matrix, and $V^{T}$ is the transpose of $\mathbf{V}$. The diagonal elements of $\mathbf{S}$ satisfy $\sigma_{1} \geq \cdots \geq \sigma_{r} \geq \sigma_{r+1}=\cdots=$ $\sigma_{n}=0$, where $r$ is the rank of AA and is equal to the number of nonnegative singular values.

An important property of SVD is that the singular values (SVs) of an image possess significant stability because these values do not vary rapidly with small image perturbations. Therefore, a watermark embedded in a singular matrix exhibits high robustness [19-23].

\section{Proposed Watermarking Scheme}

3.1. Texture Directionality. Texture is a property that exists in many areas. In the scope of image processing, texture denotes a trend or pattern that reflects a change in the intensity and direction of image pixels. Directionality is one of the most significant characteristic features. This paper proposes a method based on statistical measurements for calculating image directionality from the directional histogram [24, 25].

For a given image pixel, if the outputs of the horizontal and vertical edge operators are $\Delta H$ and $\Delta V$, respectively, then the corresponding gradient vector $\Delta G$ is

$$
\begin{aligned}
|\Delta G| & =\frac{|\Delta H|+|\Delta V|}{2} \\
\theta & =\frac{\pi}{2}+\tan ^{-1}\left(\frac{\Delta V}{\Delta H}\right) \quad 0 \leq \theta \leq 180^{\circ} .
\end{aligned}
$$

Thus, the modulus $|\Delta G|$ and angle $\theta$ of the vector are obtained. The direction edge histogram $H_{d}$ is then calculated by quantizing $\theta$ and counting the ratio of effective pixels. A direction edge histogram for the Lena test image is shown in Figure 3(a).

Assuming that the variation trend denotes $n$ peaks in the histogram, Figure 3(b) shows one of these peaks. Let $w_{c}$ be a collection of bins from the previous valley $\phi_{1}$ to the next valley $\phi_{2}$ for each peak $p$, and let $\lambda_{p}$ be the angle position of the peak. $H_{d}(\lambda)$ is the bin height at angle position $\lambda$. Texture directionality is calculated as [24]

$$
f_{\text {dir }}=\sum_{p}^{n} \sum_{\lambda \in w_{c}}\left(\lambda-\lambda_{p}\right)^{2} H_{d}(\lambda)
$$

The image texture direction is computed as a numerical value based on the above description. Figure 4 shows reconstructed images for eight shearlet direction coefficients, and the corresponding texture directionality values are shown in Table 1 . The fourth subband with the largest value represents 


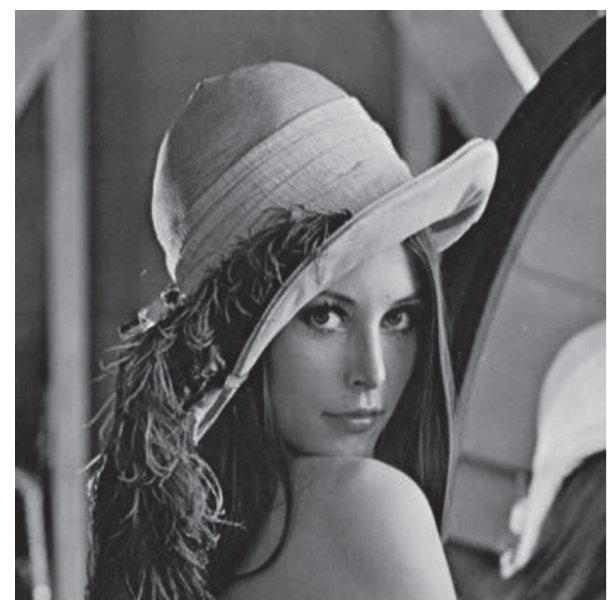

(a)

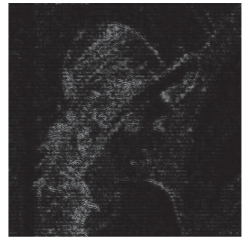

D1

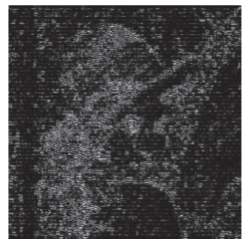

D5

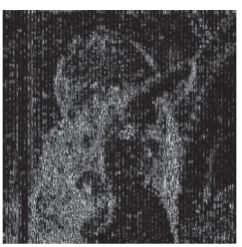

D2

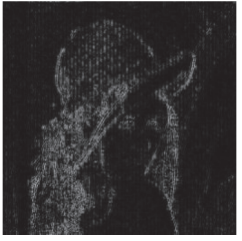

D6

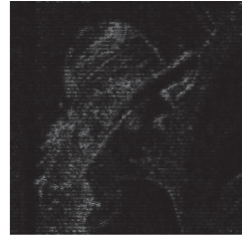

D3

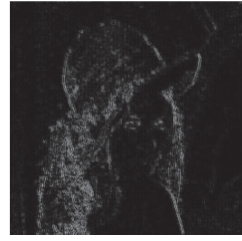

D7

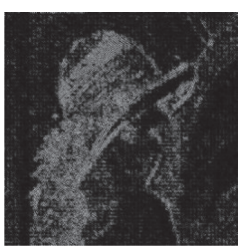

D4

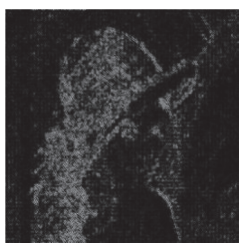

D8

(b)

Figure 4: (a) Lena test image. (b) Eight reconstructed images (D1-D8) of shearlet direction coefficients.

strong directionality and is selected to carry the watermark. The texture of an image with strong directionality reflects the contours and directional edges, and the directional filter bank of NSST can effectively capture high-frequency content. Further, due to its scale-invariant nature, stability does not change with scaling, rotation, or noise attacks. Thus, this property is an advantage for watermark hiding and robustness.

3.2. Proposed Watermarking Algorithm. Consider that the cover (host) image is a grayscale image $f(i, j)$ with size $N \times N$, while the watermark is a binary image $\mathbf{W}$ with size $M \times M$ and elements $w(i, j)$. The selection of a suitable location, effective coefficients to embed, and the embedding mechanism is of crucial importance in any watermarking scheme. In the proposed scheme, we select the eighth directional subband of level 3 with maximum directionality. This suggests that the subband has significant values to indicate the presence of directional edges, which contribute to watermark imperceptibility. Details on the embedding and extraction process are provided below.

\subsubsection{Watermark Embedding}

Step 1. In order to increase watermarking security, the encrypted watermark $W^{*}$ is obtained through the Arnold scrambling transform:

$$
W^{*}=\operatorname{arnold}(W, \mathrm{key}),
$$

where key represents scrambling times and is held to the decrypted watermark as the secret key.

Step 2. A host image with size $512 \times 512$ is decomposed by 2 -level NSST. An approximation image and directional subbands $S_{i, j}\left(1 \leq i \leq 2,1 \leq j \leq 2^{k_{i}}\right)$ with size $512 \times 512$ are obtained. 
TABLE 2: The relationship between $\alpha$ and watermarking imperceptibility.

\begin{tabular}{lccccc}
\hline$\alpha$ & 0.05 & 0.2 & 0.5 & 0.7 & 1 \\
\hline SSIM & 1.0000 & 1.0000 & 0.9999 & 0.9998 & 0.9973 \\
\hline
\end{tabular}

Step 3. The subband with maximum texture directionality is divided into nonoverlapping blocks of size $64 \times 64$, equal to the size of the watermark.

Step 4. SVD is performed on the block that has the largest energy $L^{8}$, and the singular value matrix $S$ of size $64 \times 64$ is obtained to achieve watermark embedding based on the rule

$$
\begin{aligned}
{\left[\begin{array}{lll}
U & S & V
\end{array}\right] } & =\operatorname{SVD}(L), \\
S S & =S+\alpha * W^{*},
\end{aligned}
$$

where SS represents the singular value matrix containing watermarking information and $\alpha$ is the embedding factor used to control imperceptibility. With an increasing value of $\alpha$, the amount of the embedded watermark is increased, which means that the values of SSIM (see (14)) become larger and that the distortion is more pronounced. Table 2 shows the relationship between values of $\alpha$ and the watermark imperceptibility in terms of SSIM.

Step 5. The watermarked image $I^{\prime}$ is obtained by the recombination of modified singular values and inverse NSST.

3.2.2. Watermark Extraction. Watermark extraction is the inverse process of watermark embedding. Note that the original image is used here to extract the watermark. The steps of watermark extraction are provided below.

Step 1. A 2-level NSST is applied to the watermarked image.

Step 2. The subband with maximum directionality is divided into nonoverlapping blocks.

Step 3. SVD is applied to the block with the largest energy $L^{\prime}$, and the singular value matrix $S S^{\prime}$ is obtained.

Step 4. The singular value matrix $S$ is used to acquire the encrypted watermark $\widehat{W}^{*}$ as follows:

$$
\widehat{W}^{*}=\frac{\left(S S^{\prime}-S\right)}{\alpha} \text {. }
$$

Step 5. The extracted watermark $W^{\prime}$ is obtained by the inverse Arnold transform in combination with key:

$$
W^{\prime}=\operatorname{iarnold}\left(\widehat{W}^{*}, \text { key }\right)
$$

\section{Experimental Analysis}

A large number of varied test images that reflect different scenes were used to test the effectiveness of the scheme. Lena, Snow, Snow2, Plane, Baboon, Woman, Lake, Crowd, Peppers,
Scenery, Man, and Bridge (each with size $512 \times 512$ ) were selected as host images and a binary logo with size $64 \times 64$ was selected as the watermark (Figure 5). The "pkva" filters of NSST are used with the number of directional subbands set as 8 and 16. Also, the values of $\alpha$ and the secret key are set to 0.7 and 10, respectively, after numerous experimental trials. Structural similarity index measurement (SSIM) is used to measure visual quality and watermark robustness is assessed by normalized correlation (NC) and bit error rate (BER).

$$
\operatorname{SSIM}(x, y)=\frac{\left(2 \mu_{x} \mu_{y}+C_{1}\right)\left(2 \sigma_{x y}+C_{2}\right)}{\left(\mu_{x}^{2}+\mu_{y}^{2}+C_{1}\right)\left(\sigma_{x}^{2}+\sigma_{y}^{2}+C_{2}\right)},
$$

where $\mu_{x}$ and $\mu_{y}$ are the averages of $x$ and $y, \sigma_{x}^{2}$ and $\sigma_{y}^{2}$ are the variances of $x$ and $y$, and $\sigma_{x y}$ is the covariance of $x$ and $y$.

$$
\begin{aligned}
\operatorname{NC}\left(w, w^{\prime}\right) \\
=\frac{\sum_{i=0}^{M} \sum_{j=0}^{M} w(i, j) * w^{\prime}(i, j)}{\sqrt{\sum_{i=0}^{M} \sum_{j=0}^{M} w(i, j)} \sqrt{\sum_{i=0}^{M} \sum_{j=0}^{M} w^{\prime}(i, j)}},
\end{aligned}
$$

where $w(i, j)$ represents the elements of the original watermark and $w^{\prime}(i, j)$ represents the elements of the extracted watermark.

$$
\mathrm{BER}=\frac{B}{P \times Q} \times 100 \%,
$$

where $B$ is the number of erroneously detected bits for a $P \times Q$ watermark image.

4.1. Watermarking Invisibility. The performance of the proposed algorithm was tested under no attacks. Watermark imperceptibility in terms of SSIM is shown in Table 3. SSIM values between the host and watermarked images for the proposed algorithm are greater than 0.99 and reached 1 in some images, which shows good watermark invisibility. Further, the watermark can be extracted with little distortion. NC values of the extracted watermarks are greater than 0.99 and BER values are near 0 , which illustrates effective watermark extraction.

4.2. Watermarking Robustness. Tables 4-9 list the extracted watermarks for various attacks (scaling, JPEG compression, noise, and filtering) and the corresponding NC and BER values. Results show high robustness against various attacks, where the average $\mathrm{NC}$ and BER values are found to reach 0.985 and 0.37 , respectively. The watermarking algorithm does not exhibit strong robustness for average filtering attack $(8 * 8)$ and rotation attack, for which the average NC values are 0.96 and 0.95 , respectively. The performance of the watermark for various attacks is analyzed in detail in the following subsections.

4.2.1. Robustness to Scaling Attacks. Scaling operations can be used to attack a watermarked image. Table 3 shows the values of SSIM, NC, and BER for scaling attacks with scaling parameters $1 / 4,1 / 2,2$, and 4 . Figure 6 shows the extracted watermark from the attacked images (Snow, Plane, and Crowd) for a scaling parameter of $1 / 2$. 


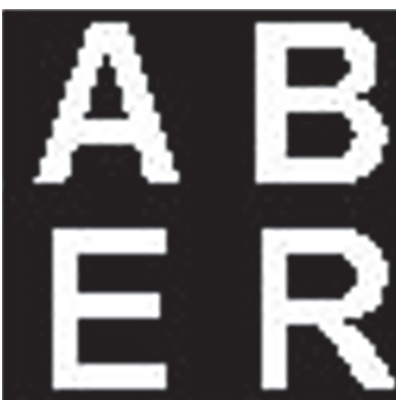

(a)

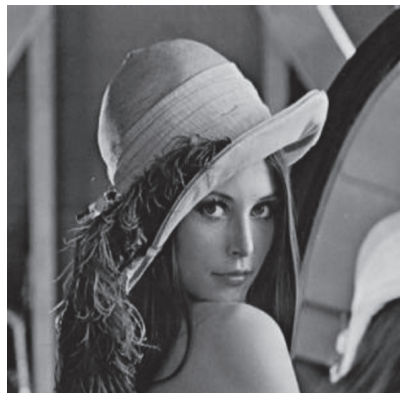

(b)

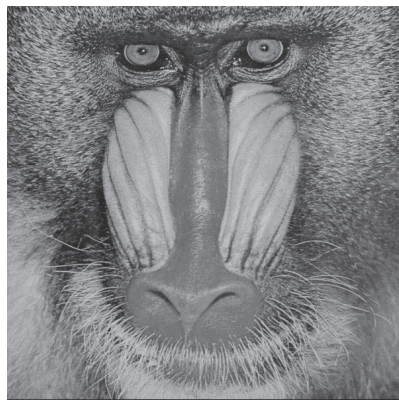

(f)

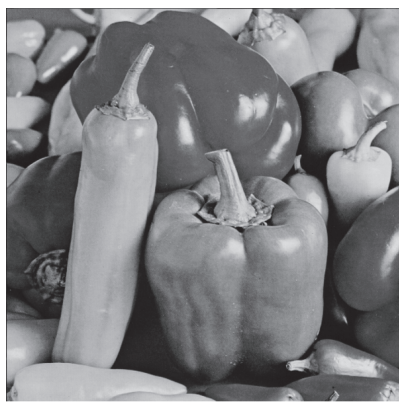

(j)

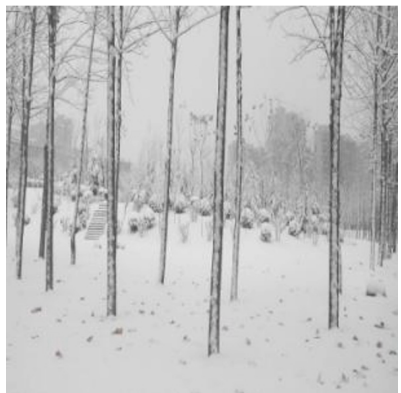

(c)

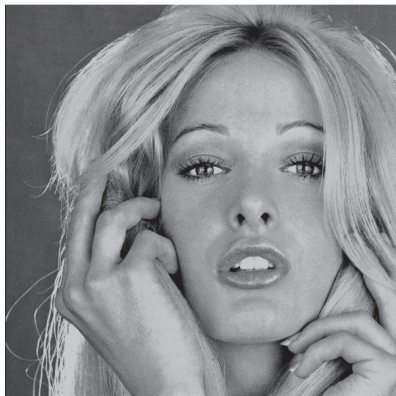

(g)

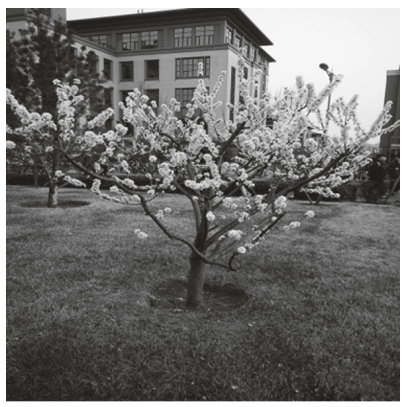

(k)

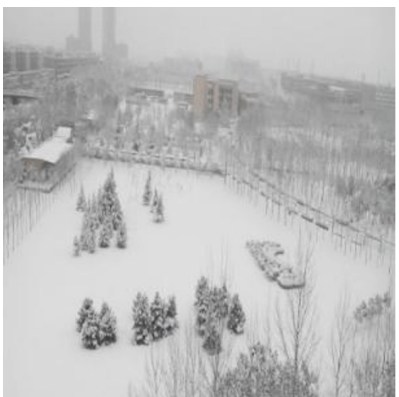

(d)

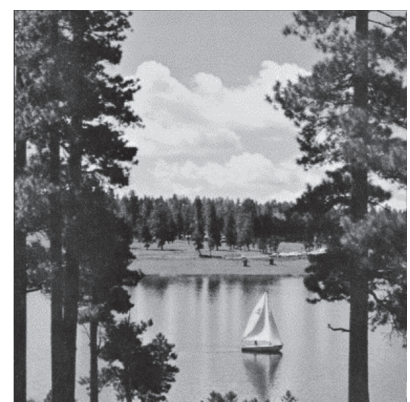

(h)

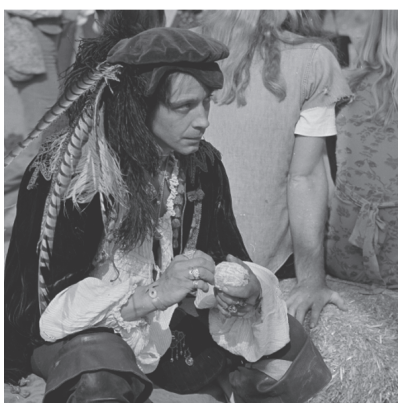

(l)

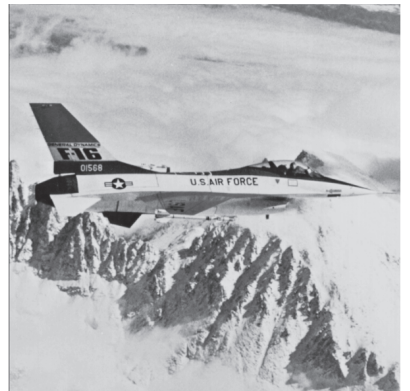

(e)

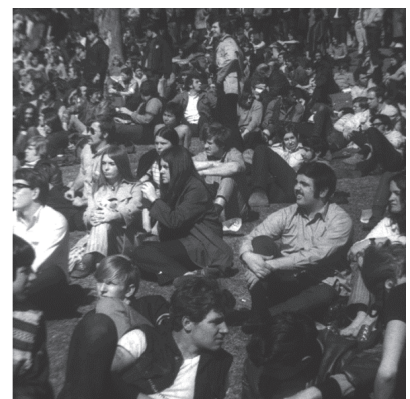

(i)

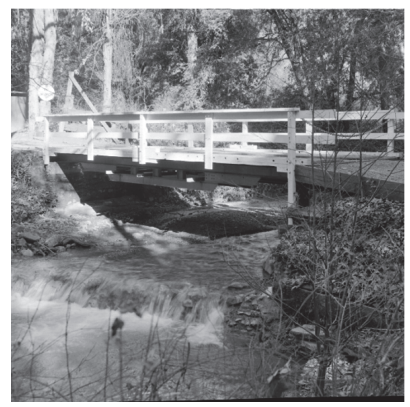

(m)

Figure 5: Original images: (a) watermark image, (b) Lena, (c) Snow, (d) Snow2, (e) Plane, (f) Baboon, (g) Woman, (h) Lake, (i) Crowd, (j) Peppers, (k) Scenery, (l) Man, and (m) Bridge.

For these test images, the extracted watermarks are clearly distinguishable. NC is greater than 0.98 and average BER is 0.8 . In comparison with the other images, the result for Crowd exhibits limited robustness, but its NC value reaches 0.96 , and the watermark can be extracted clearly. Therefore, the proposed algorithm can resist scaling attacks.
4.2.2. Robustness to JPEG Compression Attacks. JPEG compression is a commonly used attack in image processing. Table 5 shows the values of NC and BER for JPEG compression with different quality factors. Results suggest that, irrespective of compression factor, $\mathrm{NC}$ values are greater than 0.94. Therefore, the proposed algorithm is robust against 
TABLE 3: Experimental results of invisibility.

\begin{tabular}{l} 
Lena $\mathrm{SSIM}=0.9997$ \\
\hline
\end{tabular}

TABLE 4: Experimental results for the scaling attack.

\begin{tabular}{lcccc}
\hline Test image & Scale $=1 / 4$ & Scale $=1 / 2$ & Scale $=2$ & Scale $=4$ \\
SSIM/NC/BER & SSIM/NC/BER & SSIM/NC/BER & $0.9893 / 0.9946 / 0.2686$ \\
Lena & $0.9599 / 0.9829 / 0.8545$ & $0.9178 / 0.9702 / 1.4893$ & $0.9888 / 0.9946 / 0.2686$ & $0.9764 / 0.9937 / 0.3174$ \\
Snow & $0.9056 / 0.9956 / 0.2197$ & $0.8074 / 0.9961 / 0.1953$ & $0.9752 / 0.9951 / 0.2441$ & $0.9794 / 0.9829 / 0.8545$ \\
Snow2 & $0.9138 / 0.9985 / 0.0732$ & $0.8248 / 0.9902 / 0.4883$ & $0.9783 / 0.9985 / 0.0732$ & $0.9962 / 0.9834 / 0.8301$ \\
Crowd & $0.9831 / 0.9604 / 1.9775$ & $0.9469 / 0.9902 / 0.4883$ & $0.9960 / 0.9648 / 1.7578$ & $0.9803 / 0.9951 / 0.2441$ \\
Woman & $0.9229 / 0.9897 / 0.5127$ & $0.8451 / 0.9937 / 0.3174$ & $0.9793 / 0.9946 / 0.2686$ & $0.9402 / 0.9922 / 0.3906$ \\
Lake & $0.8009 / 0.9897 / 0.5127$ & $0.6969 / 0.9863 / 0.6836$ & $0.9373 / 0.9917 / 0.4150$ & $0.9925 / 0.9634 / 1.8311$ \\
Plane & $0.9382 / 0.9785 / 1.0742$ & $0.8480 / 0.9946 / 0.2686$ & $0.9851 / 0.9834 / 0.8301$ & $0.9523 / 0.9985 / 0.0732$ \\
Baboon & $0.8145 / 0.9961 / 0.1953$ & $0.6419 / 0.9946 / 0.2686$ & $0.9498 / 0.9829 / 0.8545$ & $0.9707 / 0.9932 / 0.3418$ \\
Peppers & $0.9131 / 0.9927 / 0.3662$ & $0.8616 / 0.9922 / 0.3906$ & $0.9694 / 0.9941 / 0.2930$ & $0.9432 / 0.9727 / 1.3672$ \\
Scenery & $0.7834 / 0.9243 / 3.7842$ & $0.6043 / 0.9097 / 4.5166$ & $0.9402 / 0.9727 / 1.3672$ & $0.9888 / 0.9912 / 0.4395$ \\
Man & $0.9526 / 0.9849 / 0.7568$ & $0.8840 / 0.9897 / 0.5127$ & $0.9882 / 0.9922 / 0.3906$ & $0.9811 / 0.9888 / 0.5615$ \\
Bridge & $0.9227 / 0.9160 / 4.1992$ & $0.8152 / 0.9858 / 0.7080$ & $0.9820 / 0.9780 / 1.0986$ \\
\hline
\end{tabular}

JPEG compression. Three perfectly extracted watermarks from a JPEG attack (quality factor $Q=40$ ) are shown in Figure 7.

4.2.3. Robustness to Noise Attacks. In order to evaluate the robustness of the proposed scheme against noise, every watermarked image is attacked by "Gaussian" and "Salt and Pepper" noise with variances of $0.001,0.005,0.01$, and 0.05 . Results are shown in Tables 6 and 7. For a "Gaussian" noise attack, noise points are found to appear in the extracted watermarks when the noise variance increases. However, the extracted watermark is still distinguishable in all test images.

Similarly, for a "Salt and Pepper" noise attack, the algorithm shows good robustness. BER is 0 for Woman and Lake with a noise variance of 0.001 , which illustrates tremendous robustness to noise attacks. Figures 8 and 9 show watermarks extracted from images attacked using "Gaussian" and "Salt and Pepper" noise with a variance of 0.001 . 
TABLE 5: Experimental results for the JPEG compression attack.

\begin{tabular}{|c|c|c|c|c|}
\hline Test image & $\begin{array}{c}\text { JPEG }=30 \\
\text { SSIM/NC/BER }\end{array}$ & $\begin{array}{c}\text { JPEG }=40 \\
\text { SSIM/NC/BER }\end{array}$ & $\begin{array}{c}\text { JPEG }=75 \\
\text { SSIM/NC/BER }\end{array}$ & $\begin{array}{c}\text { JPEG }=90 \\
\text { SSIM/NC/BER }\end{array}$ \\
\hline Lena & $0.8752 / 0.9736 / 1.3184$ & $0.8914 / 0.9751 / 1.2451$ & $0.9293 / 0.9937 / 0.3174$ & $0.9552 / 0.9946 / 0.2686$ \\
\hline Snow & $0.8477 / 0.9932 / 0.3418$ & $0.8706 / 0.9980 / 0.0977$ & $0.9262 / 0.9902 / 0.4883$ & $0.9806 / 0.9941 / 0.2930$ \\
\hline Snow2 & $0.8483 / 0.9697 / 1.5137$ & $0.8723 / 0.9985 / 0.0732$ & $0.9274 / 0.9985 / 0.0732$ & $0.9801 / 0.9990 / 0.0488$ \\
\hline Crowd & $0.9156 / 0.9692 / 1.5381$ & $0.9321 / 0.9741 / 1.2939$ & $0.9658 / 0.9937 / 0.3174$ & $0.9834 / 0.9688 / 1.5625$ \\
\hline Woman & 0.8191/0.9790/1.0498 & $0.8416 / 0.9854 / 0.7324$ & $0.9041 / 0.9829 / 0.8545$ & $0.9480 / 0.9937 / 0.3174$ \\
\hline Lake & $0.6994 / 0.9902 / 0.4883$ & $0.7213 / 0.9907 / 0.4639$ & $0.8199 / 1.0000 / 0.0000$ & $0.9488 / 0.9858 / 0.7080$ \\
\hline Plane & $0.8834 / 0.9409 / 2.9541$ & $0.9024 / 0.9458 / 2.7100$ & $0.9432 / 0.9688 / 1.5625$ & $0.9666 / 0.9717 / 1.4160$ \\
\hline Baboon & $0.7598 / 0.9976 / 0.1221$ & $0.7905 / 0.9976 / 0.1221$ & $0.8777 / 0.9028 / 4.8548$ & $0.9748 / 0.9995 / 0.0244$ \\
\hline Peppers & $0.8158 / 0.9600 / 2.0020$ & $0.8336 / 0.9785 / 1.0742$ & $0.8771 / 0.9863 / 0.6836$ & $0.9227 / 0.9932 / 0.3418$ \\
\hline Scenery & $0.7682 / 0.9546 / 2.2705$ & $0.8117 / 0.9761 / 1.1963$ & $0.9334 / 0.9780 / 1.0986$ & $0.9837 / 0.9897 / 0.5127$ \\
\hline Man & $0.8646 / 0.9844 / 0.7813$ & $0.8857 / 0.9863 / 0.6836$ & $0.9364 / 0.9917 / 0.4150$ & $0.9682 / 0.9922 / 0.3906$ \\
\hline Bridge & $0.8650 / 0.9546 / 2.2705$ & $0.8868 / 0.9619 / 1.9043$ & $0.9435 / 0.9717 / 1.4160$ & 0.9795/0.9780/1.0986 \\
\hline
\end{tabular}

TABLE 6: Experimental results for the "Gaussian" white noise attack.

\begin{tabular}{lcccc}
\hline Test image & Var $=0.001$ & Var $=0.005$ & Var $=0.01$ & Var $=0.05$ \\
SSIM/NC/MCR & SSIM/NC/BER & $0.0989 / 0.9907 / 0.4639$ \\
\hline Lena & $0.6183 / 0.9960 / 0.2209$ & $0.3432 / 0.9951 / 0.2441$ & $0.2481 / 0.9932 / 0.3418$ & $0.3709 / 0.9751 / 1.2451$ \\
Snow & $0.6721 / 0.9941 / 0.2930$ & $0.4617 / 0.9897 / 0.5127$ & $0.3710 / 0.98191 / 0.9033$ & $0.1009 / 0.9883 / 0.5859$ \\
Snow2 & $0.6239 / 0.9985 / 0.0732$ & $0.3641 / 0.9966 / 0.1709$ & $0.2643 / 0.9912 / 0.4395$ & $0.1869 / 0.9941 / 0.2930$ \\
Crowd & $0.7400 / 0.9971 / 0.1465$ & $0.5013 / 0.9956 / 0.2197$ & $0.3962 / 0.9951 / 0.2441$ & $0.1217 / 0.9893 / 0.5371$ \\
Woman & $0.6985 / 0.9980 / 0.0977$ & $0.4140 / 0.9961 / 0.1953$ & $0.3029 / 0.9951 / 0.2441$ & $0.2147 / 0.9917 / 0.4150$ \\
Lake & $0.8259 / 0.9946 / 0.2686$ & $0.5657 / 0.9937 / 0.3174$ & $0.4452 / 0.9922 / 0.3906$ & $0.9925 / 0.9634 / 1.8311$ \\
Plane & $0.6115 / 0.9785 / 1.0742$ & $0.8480 / 0.9946 / 0.2686$ & $0.9851 / 0.9834 / 0.8301$ & $0.1389 / 0.9805 / 0.9766$ \\
Baboon & $0.8925 / 0.9858 / 0.7080$ & $0.3671 / 0.9854 / 0.7324$ & $0.2853 / 0.9814 / 0.9277$ & $0.1190 / 0.9717 / 1.4160$ \\
Peppers & $0.6661 / 0.9966 / 0.1709$ & $0.3805 / 0.9902 / 0.4883$ & $0.2802 / 0.9897 / 0.5127$ & $0.3370 / 0.9780 / 1.0986$ \\
Scenery & $0.8961 / 0.9961 / 0.1953$ & $0.7407 / 0.9956 / 0.2197$ & $0.6310 / 0.9883 / 0.5859$ & $0.1454 / 0.9917 / 0.4150$ \\
Man & $0.7385 / 0.9951 / 0.2441$ & $0.4691 / 0.9941 / 0.2930$ & $0.3514 / 0.9922 / 0.3906$ & $0.2471 / 0.9717 / 1.4160$ \\
Bridge & $0.8812 / 0.9980 / 0.0977$ & $0.6653 / 0.9961 / 0.1953$ & $0.5342 / 0.9951 / 0.2441$ & \\
\hline
\end{tabular}

TABLE 7: Experimental results for the "Salt and Pepper" noise attack.

\begin{tabular}{lcccc}
\hline Test image & $\begin{array}{c}\text { Var }=0.001 \\
\text { SSIM/NC/BER }\end{array}$ & $\begin{array}{c}\text { Var }=0.005 \\
\text { SSIM/NC/BER }\end{array}$ & $\begin{array}{c}\text { Var }=0.01 \\
\text { SSIM/NC/BER }\end{array}$ & $\begin{array}{c}\text { Var }=0.05 \\
\text { SSIM/NC/BER }\end{array}$ \\
\hline Lena & $0.9634 / 0.9961 / 0.1953$ & $0.8350 / 0.9951 / 0.2441$ & $0.6992 / 0.9927 / 0.3662$ & $0.2601 / 0.9893 / 0.5371$ \\
Snow & $0.9750 / 0.9995 / 0.0244$ & $0.8720 / 0.9941 / 0.2930$ & $0.7585 / 0.9849 / 0.7568$ & $0.3737 / 0.9795 / 1.0254$ \\
Snow2 & $0.9664 / 0.9990 / 0.0488$ & $0.8484 / 0.9985 / 0.0732$ & $0.7226 / 0.9980 / 0.0977$ & $0.2806 / 0.9976 / 0.1221$ \\
Crowd & $0.9739 / 0.9956 / 0.2197$ & $0.8822 / 0.9941 / 0.2930$ & $0.7835 / 0.9893 / 0.5371$ & $0.4001 / 0.9727 / 1.3672$ \\
Woman & $0.9669 / 1.0000 / 0.0000$ & $0.8406 / 0.9985 / 0.0732$ & $0.7232 / 0.9951 / 0.2441$ & $0.3120 / 0.9946 / 0.2686$ \\
Lake & $0.9799 / 1.0000 / 0.0000$ & $0.8931 / 0.9927 / 0.3662$ & $0.8074 / 0.9771 / 1.1475$ & $0.4327 / 0.9771 / 1.1475$ \\
Plane & $0.9630 / 0.9990 / 0.0488$ & $0.8441 / 0.9985 / 0.0732$ & $0.7197 / 0.9829 / 0.8545$ & $0.3028 / 0.9751 / 1.2451$ \\
Baboon & $0.9826 / 0.9995 / 0.0244$ & $0.9146 / 0.9868 / 0.6592$ & $0.8416 / 0.9849 / 0.7568$ & $0.5131 / 0.9834 / 0.8301$ \\
Peppers & $0.9597 / 0.9990 / 0.0488$ & $0.8392 / 0.9941 / 0.2930$ & $0.7190 / 0.9897 / 0.5127$ & $0.2929 / 0.9795 / 1.0254$ \\
Scenery & $0.9860 / 0.9937 / 0.3174$ & $0.9363 / 0.9888 / 0.5615$ & $0.8779 / 0.9600 / 2.0020$ & $0.5842 / 0.9580 / 2.0996$ \\
Man & $0.9685 / 0.9985 / 0.0732$ & $0.8655 / 0.9956 / 0.2197$ & $0.7591 / 0.9922 / 0.3906$ & $0.3522 / 0.9766 / 1.1719$ \\
Bridge & $0.9816 / 0.9956 / 0.2197$ & $0.9150 / 0.9888 / 0.5615$ & $0.8383 / 0.9785 / 1.0742$ & $0.5042 / 0.9717 / 1.4160$ \\
\hline
\end{tabular}


TABLE 8: Experimental results for the filtering attack.

\begin{tabular}{|c|c|c|c|c|}
\hline Test image & $\begin{array}{l}\text { Average filtering } \\
\quad(4 * 4) \\
\text { SSIM/NC/BER }\end{array}$ & $\begin{array}{l}\text { Average filtering } \\
\quad(8 * 8) \\
\text { SSIM/NC/BER }\end{array}$ & $\begin{array}{l}\text { Median filtering } \\
(3 * 3) \\
\text { SSIM/NC/BER }\end{array}$ & $\begin{array}{l}\text { Median filtering } \\
(4 * 4) \\
\text { SSIM/NC/BER }\end{array}$ \\
\hline Lena & $0.8963 / 0.9897 / 0.5127$ & $0.6256 / 0.9888 / 0.5615$ & $0.9171 / 0.9893 / 0.5371$ & $0.8560 / 0.9927 / 0.3662$ \\
\hline Snow & 0.7918/0.9937/0.3174 & $0.5378 / 0.9849 / 0.7568$ & $0.8142 / 0.9946 / 0.2686$ & $0.7938 / 0.9854 / 0.7324$ \\
\hline Snow2 & $0.8140 / 0.9956 / 0.2197$ & $0.5604 / 0.9795 / 1.0254$ & $0.8022 / 0.9985 / 0.0732$ & $0.7287 / 0.9976 / 0.1221$ \\
\hline Crowd & $0.9528 / 0.9517 / 2.4170$ & $0.6273 / 0.9517 / 2.4170$ & $0.9581 / 0.9717 / 1.4160$ & $0.8739 / 0.9937 / 0.3174$ \\
\hline Woman & $0.8149 / 0.9888 / 0.5615$ & $0.5439 / 0.9878 / 0.6104$ & $0.8450 / 0.9946 / 0.2686$ & $0.9803 / 0.9951 / 0.2441$ \\
\hline Lake & $0.5161 / 0.9604 / 1.9775$ & $0.3495 / 0.9580 / 2.0996$ & $0.6287 / 0.9907 / 0.4639$ & $0.6084 / 0.9917 / 0.4150$ \\
\hline Plane & $0.9335 / 0.9990 / 0.0488$ & $0.6509 / 0.9736 / 1.3184$ & $0.9391 / 0.9985 / 0.0732$ & $0.8734 / 0.9619 / 1.9043$ \\
\hline Baboon & $0.5440 / 0.9966 / 0.1709$ & $0.3143 / 0.9858 / 0.7080$ & $0.6062 / 0.9971 / 0.1465$ & $0.4693 / 0.9951 / 0.2441$ \\
\hline Peppers & $0.7599 / 0.9858 / 0.7080$ & $0.5429 / 0.9795 / 1.0254$ & $0.8605 / 0.9941 / 0.2930$ & $0.8082 / 0.9937 / 0.3174$ \\
\hline Scenery & $0.4698 / 0.9600 / 2.0020$ & $0.2828 / 0.9590 / 2.0508$ & $0.5736 / 0.9897 / 0.5127$ & $0.4622 / 0.9727 / 1.3672$ \\
\hline Man & $0.8802 / 0.9834 / 0.8301$ & $0.5707 / 0.9785 / 1.0742$ & $0.8957 / 0.9946 / 0.2686$ & $0.7916 / 0.9546 / 2.2705$ \\
\hline Bridge & $0.8086 / 0.9771 / 1.1475$ & $0.4776 / 0.9717 / 1.4160$ & $0.8205 / 0.9937 / 0.3174$ & $0.6796 / 0.9907 / 0.4639$ \\
\hline
\end{tabular}

TABLE 9: Experimental results for the rotation attack.

\begin{tabular}{lcccc}
\hline Test image & $\begin{array}{c}\text { Angle }=5^{\circ} \\
\text { SSIM/NC/BER }\end{array}$ & $\begin{array}{c}\text { Angle }=10^{\circ} \\
\text { SSIM/NC/BER }\end{array}$ & $\begin{array}{c}\text { Angle }=15^{\circ} \\
\text { SSIM/NC/BER }\end{array}$ & $\begin{array}{c}\text { Angle }=30^{\circ} \\
\text { SSIM/NC/BER }\end{array}$ \\
\hline Lena & $0.3521 / 0.9907 / 0.4639$ & $0.2733 / 0.9795 / 1.0254$ & $0.2501 / 0.9624 / 1.8799$ & $0.2200 / 0.9473 / 2.6367$ \\
Snow & $0.2879 / 0.9717 / 1.4160$ & $0.2565 / 0.9702 / 1.4893$ & $0.2301 / 0.9688 / 1.5625$ & $0.1901 / 0.9639 / 1.8066$ \\
Snow2 & $0.3586 / 0.9736 / 1.3184$ & $0.3025 / 0.9639 / 1.8066$ & $0.2736 / 0.9517 / 2.4170$ & $0.2141 / 0.9409 / 2.9541$ \\
Crowd & $0.1649 / 0.9814 / 0.9277$ & $0.1191 / 0.9800 / 1.0010$ & $0.1102 / 0.9692 / 1.5381$ & $0.0822 / 0.9404 / 2.9785$ \\
Woman & $0.2764 / 0.9902 / 0.4883$ & $0.1928 / 0.9863 / 0.6836$ & $0.1554 / 0.9727 / 1.3672$ & $0.1440 / 0.9556 / 2.2217$ \\
Lake & $0.1045 / 0.9761 / 1.1963$ & $0.0882 / 0.9741 / 1.2939$ & $0.0814 / 0.9727 / 1.3672$ & $0.0656 / 0.9390 / 3.0518$ \\
Plane & $0.3484 / 0.9810 / 0.9521$ & $0.2872 / 0.9785 / 1.0742$ & $0.2615 / 0.9673 / 1.6357$ & $0.2293 / 0.9600 / 2.002$ \\
Baboon & $0.0800 / 0.9863 / 0.6836$ & $0.0635 / 0.9814 / 0.9277$ & $0.0540 / 0.9756 / 1.2207$ & $0.0454 / 0.9546 / 2.2705$ \\
Peppers & $0.2760 / 0.9858 / 0.7080$ & $0.2076 / 0.9780 / 1.0986$ & $0.1786 / 0.9717 / 1.4160$ & $0.1453 / 0.9590 / 2.0508$ \\
Scenery & $0.0817 / 0.9863 / 0.6836$ & $0.0598 / 0.9844 / 0.7813$ & $0.0470 / 0.9814 / 0.9277$ & $0.0287 / 0.9756 / 1.2207$ \\
Man & $0.1871 / 0.9897 / 0.5127$ & $0.1435 / 0.9893 / 0.5371$ & $0.1165 / 0.9785 / 1.0742$ & $0.0994 / 0.9600 / 2.0020$ \\
Bridge & $0.0584 / 0.9961 / 0.1953$ & $0.0461 / 0.9653 / 1.7334$ & $0.0418 / 0.9580 / 2.0996$ & $0.0369 / 0.9546 / 2.2705$ \\
\hline
\end{tabular}

4.2.4. Robustness to Filtering Attacks. As shown in Table 8, for both average and median filtering, the extracted watermarks are distinguishable in the test images. Three examples are presented in Figure 10 for a $3 \times 3$ median filtering attack. SSIM values of the watermarked image suggest that the image is easily distorted by the filtering attack. However, average NC is 0.975 and BER is 0.8 , which illustrates that the proposed algorithm can resist the filtering attack.

4.2.5. Robustness to Rotation Attacks. The watermark is embedded into the most obvious directional subband. As shown by the SSIM values in Table 9, the loss of information is significant when the image is rotated. In order to illustrate the ability to resist a rotation attack, watermarked images are rotated by $5^{\circ}, 10^{\circ}, 15^{\circ}$, and $30^{\circ}$. With an increase in rotation angle, the quality of the extracted watermark is reduced. The average $\mathrm{NC}$ value is 0.95 . Compared with other forms of image attacks, the quality of the extracted watermark is poorer. Figure 11 shows watermarks extracted from images attacked using rotation attack with $15^{\circ}$.

4.3. Comparative Analysis. In this section, the proposed algorithm is compared with methods based on the discrete wavelet transform domain (DWT-SVD) [20], nonsubsample contourlet transform domain (NSCT-SVD) [12], and shearlet transform domain (ST-SVD) [17]. Tables 10 and 11 and Figures 12 and 13 show a comparison of robustness for certain attacks on the four methods. The line chart indicates that NC values of the proposed NSST-SVD technique are the largest among the three methods for noise and filtering attacks. This enhancement in performance highlights the optimal nature of the shearlet transform for digital watermarking. NC for JPEG and scaling attacks is only marginally lower for the proposed algorithm compared to the others. Overall, the proposed algorithm displays noteworthy performance. 

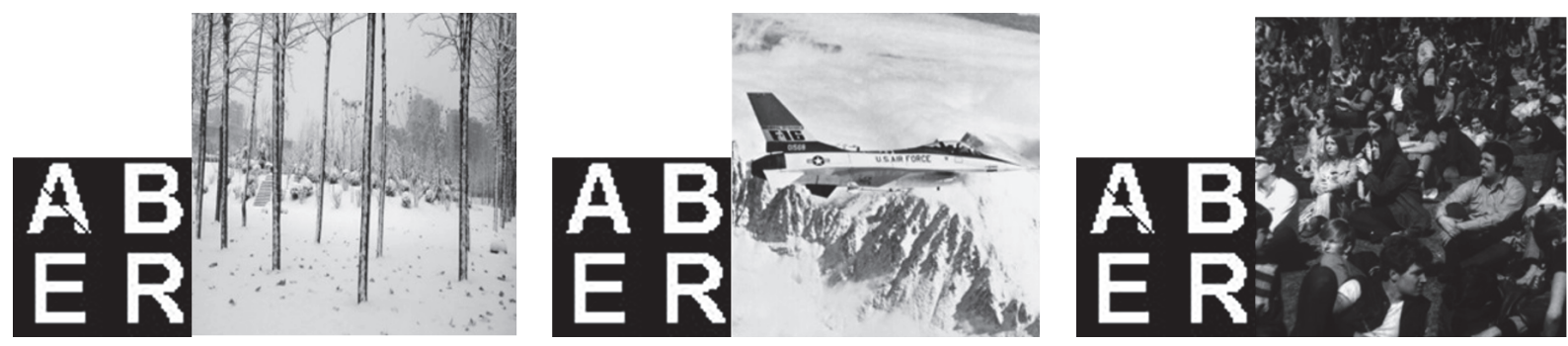

Figure 6: Extracted watermarks from attacked images (Snow, Plane, and Crowd) after a scaling attack (scale = 1/2).

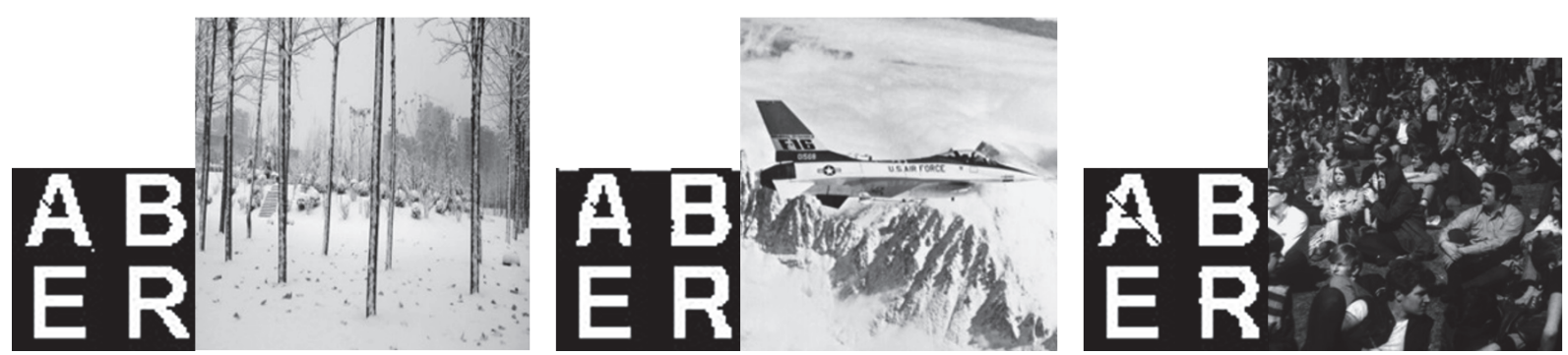

FIGURE 7: Extracted watermarks from attacked images (Snow, Plane, and Crowd) after a JPEG attack $(J P E G=40)$.

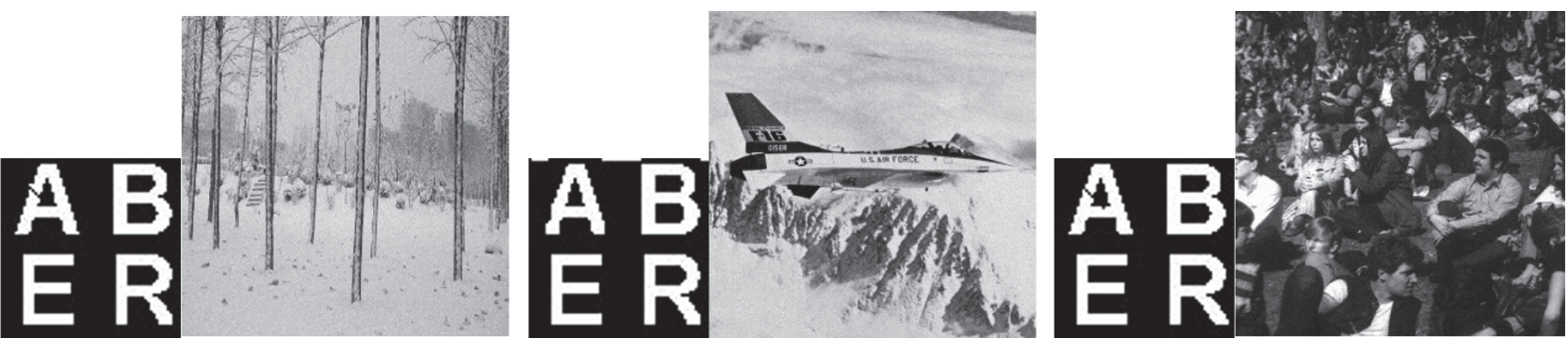

FIGURE 8: Extracted watermarks from attacked images (Snow, Plane, and Crowd) after a "Gaussian" noise attack $($ Var $=0.001)$.

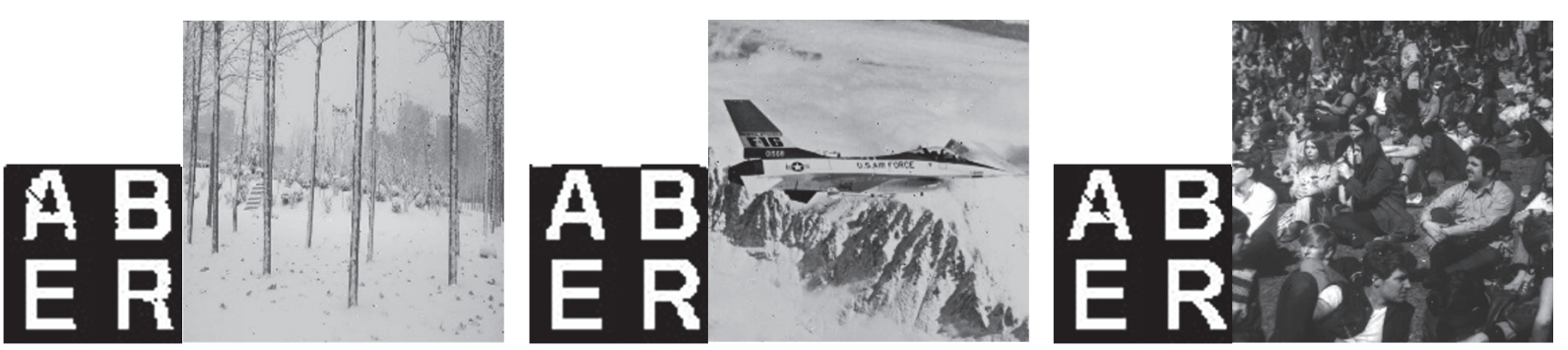

Figure 9: Extracted watermarks from attacked images (Snow, Plane, and Crowd) after a "Salt and Pepper" noise attack (Var = 0.001).

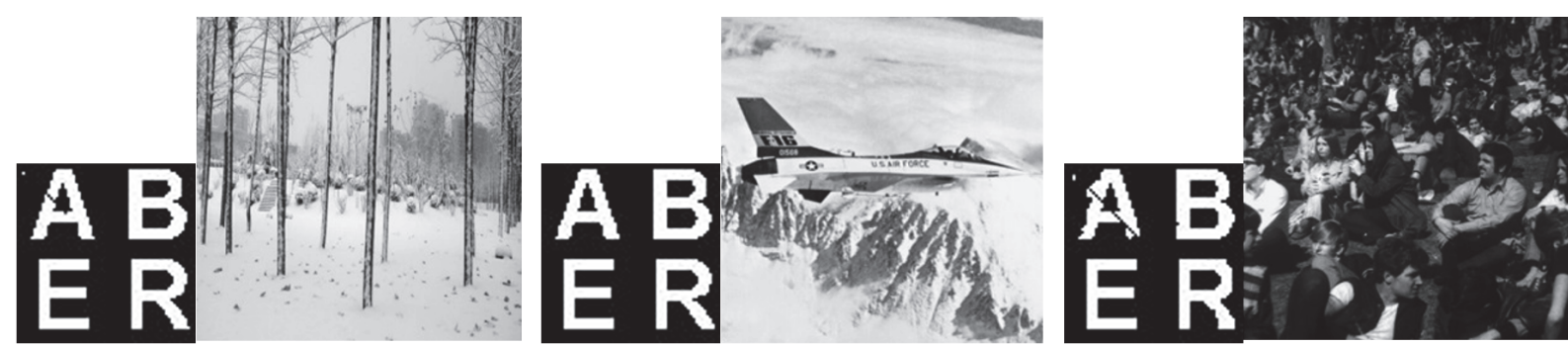

Figure 10: Extracted watermarks from attacked images (Snow, Plane, and Crowd) after a median filtering attack $(3 * 3)$. 
TABLE 10: Comparison of NC values on Lena image.

Comparison of NC values for four schemes

\begin{tabular}{|c|c|c|c|c|}
\hline Attacks & DWT-SVD & NSCT-SVD & $\begin{array}{c}\text { Ref. } \\
\text { [17]/ST-SVD }\end{array}$ & $\begin{array}{c}\text { Proposed/NSST- } \\
\text { SVD }\end{array}$ \\
\hline (1) JPEG $(Q=40)$ & 0.9971 & 1.0000 & 0.9938 & 0.9751 \\
\hline (2) Gaussian noise $($ Var $=0.001)$ & 0.7673 & 0.9683 & 0.9859 & 0.9960 \\
\hline (3) Salt and Pepper $($ Var $=0.001)$ & 0.8268 & 0.9554 & 0.9622 & 0.9961 \\
\hline (4) Scaling $($ scale $=1 / 4)$ & 0.9354 & 0.9866 & 0.9427 & 0.9829 \\
\hline (5) Average filtering $(8 * 8)$ & 0.9156 & 0.9731 & 0.9182 & 0.9888 \\
\hline (6) Median filtering $(4 * 4)$ & 0.9980 & 0.9321 & 0.9718 & 0.9927 \\
\hline
\end{tabular}

TABLE 11: Comparison of NC values on Baboon image.

\begin{tabular}{lcccc}
\hline & \multicolumn{2}{c}{ Comparison of NC values for four schemes } & & Ref. \\
Attacks & DWT-SVD & NSCT-SVD & $\begin{array}{c}\text { Proposed/NSST- } \\
\text { SVD }\end{array}$ \\
\hline (1) JPEG $(Q=40)$ & 0.9880 & 0.9983 & 0.9926 & 0.9976 \\
(2) Gaussian noise $($ Var $=0.001)$ & 0.8989 & 0.9739 & 0.9718 & 0.9858 \\
(3) Salt and Pepper $(\operatorname{Var}=0.001)$ & 0.8416 & 0.9747 & 0.9934 & 0.9995 \\
(4) Scaling (scale $=1 / 4)$ & 0.9441 & 0.9968 & 0.8742 & 0.9961 \\
(5) Average filtering $(8 * 8)$ & 0.9222 & 0.9801 & 0.8467 & 0.9858 \\
(6) Median filtering $(4 * 4)$ & 0.9803 & 0.9557 & 0.8936 & 0.9951 \\
\hline
\end{tabular}

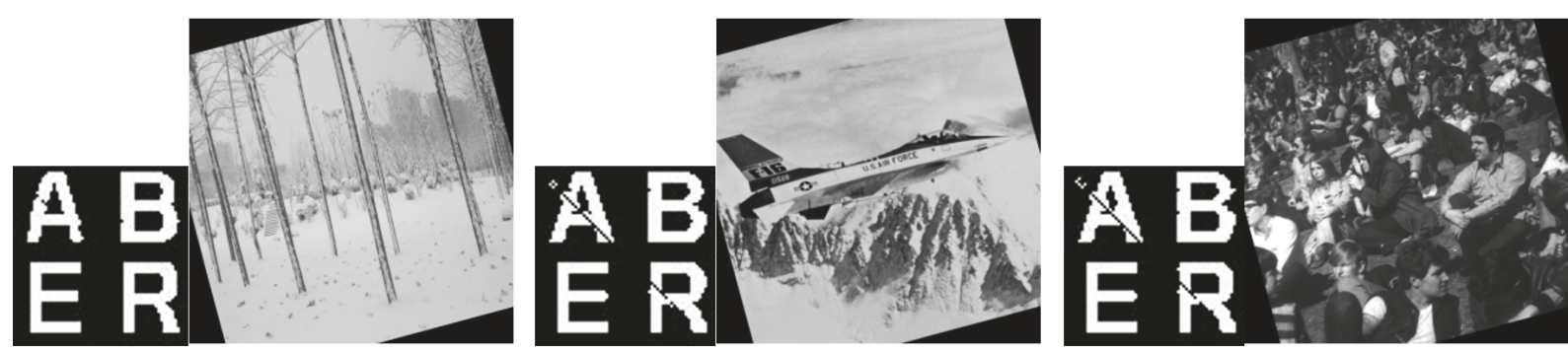

FIgURE 11: Extracted watermarks from attacked images (Snow, Plane, and Crowd) after a rotation attack $\left(15^{\circ}\right)$.

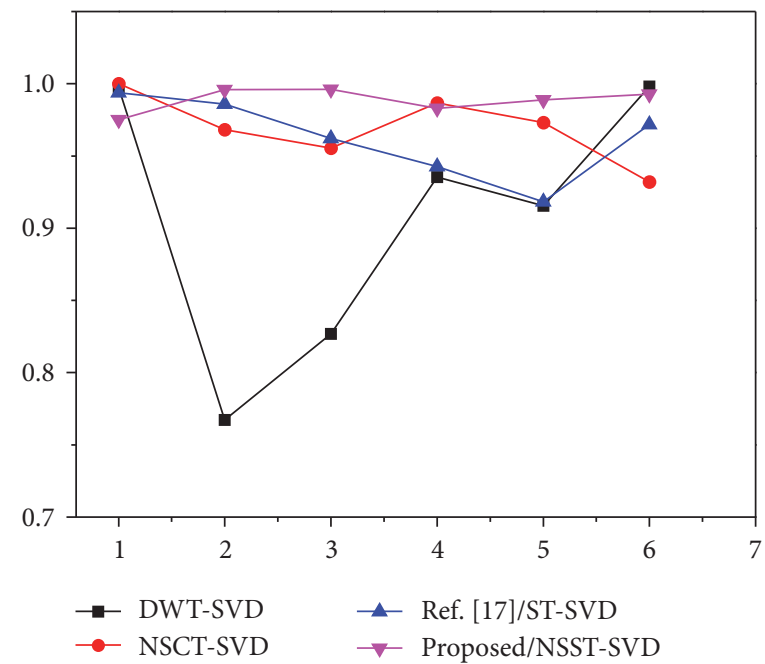

FIGURE 12: Comparison of NC for four algorithms on Lena image. 


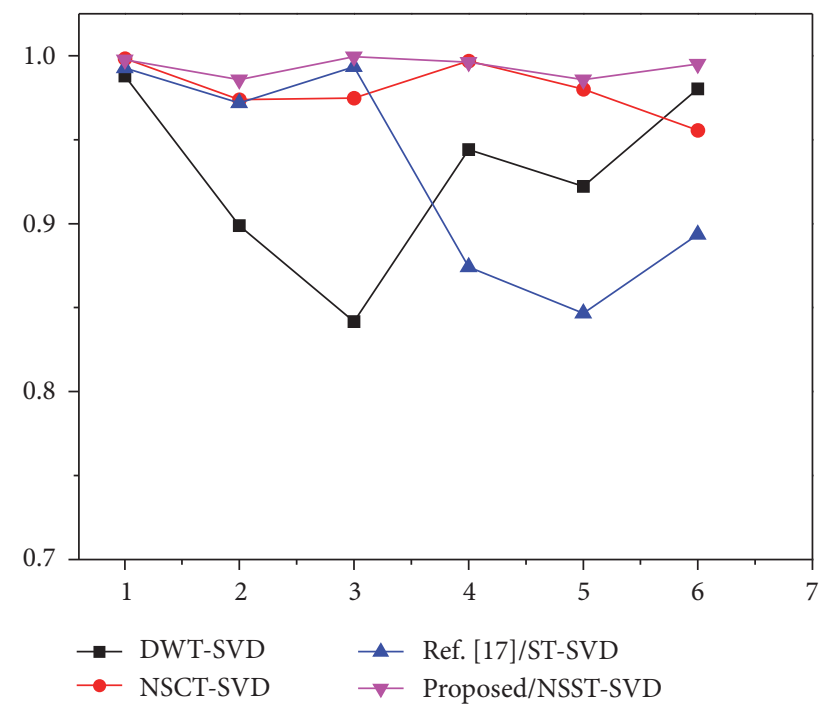

FIGURE 13: Comparison of NC for four algorithms on Baboon image.

\section{Conclusions}

A novel watermarking algorithm based on texture directionality was proposed by combining the advantages of the nonsubsample shearlet and singular value decomposition. The strongest directionality subband, which provides the most significant texture information of the image, was selected for embedding the watermark since it preserves perceptual image quality. The proposed scheme is found to exhibit noteworthy robustness for most image processing attacks such as noise, rotation, cropping, and filtering. A comparison with two existing hybrid watermarking techniques showed better performance of the proposed method for many of the tested attacks.

\section{Conflicts of Interest}

The authors declare no competing financial interests regarding the publication of this paper.

\section{Acknowledgments}

This study was financially supported by the National Natural Science Foundation of China (nos. 61379010, 61572400, and 41601353), Natural Science Basic Research Plan in Shaanxi Province of China (no. 2015JM6293), and Scientific Research Program Funded by Shaanxi Provincial Education Department (16JK1765).

\section{References}

[1] J. Fridrich, "Applications of data hiding in digital images," in Proceedings of the Fifth International Symposium on Signal Processing and Its Applications, Queensland, Australia.

[2] N. Bansal, V. K. Deolia, A. Bansal, and P. Pathak, "Digital image watermarking using least significant bit technique in different bit positions," in Proceedings of the 2014 6th International
Conference on Computational Intelligence and Communication Networks, CICN 2014, pp. 813-818, Bhopal, India, 2014.

[3] M. Cedillo-Hernandez, F. Garcia-Ugalde, M. Nakano-Miyatake, and H. Perez-Meana, "Robust object-based watermarking using SURF feature matching and DFT domain," Radioengineering, vol. 22, no. 4, pp. 1057-1071, 2013.

[4] Q. Su, G. Wang, S. Jia, X. Zhang, Q. Liu, and X. Liu, "Embedding color image watermark in color image based on two-level DCT," Signal, Image and Video Processing, vol. 9, no. 5, pp. 991-1007, 2015.

[5] A. Mishra, C. Agarwal, A. Sharma, and P. Bedi, "Optimized gray-scale image watermarkingusing DWT-SVD and firefly algorithm," Expert Systems with Applications, vol. 41, no. 17, pp. 7858-7867, 2014.

[6] M. Benouaret, A. Sahour, and S. Harize, "Real time implementation of a signal denoising approach based on eight-bits DWT," AEU-International Journal of Electronics and Communications, vol. 66, no. 11, pp. 937-943, 2012.

[7] H. Sadreazami and M. Amini, "A robust spread spectrum based image watermarking in ridgelet domain," AEU-International Journal of Electronics and Communications, vol. 66, no. 5, pp. 364-371, 2012.

[8] T. Wang and H. Li, "A novel scrambling digital image watermark algorithm based on double transform domains," Mathematical Problems in Engineering, vol. 2015, Article ID 937432, 13 pages, 2015.

[9] H. Bi, Y. Liu, M. Wu, and Y. Ge, "NSCT domain additive watermark detection using RAO hypothesis test and cauchy distribution," Mathematical Problems in Engineering, vol. 2016, Article ID 4065215, 18 pages, 2016.

[10] J. Liu, G. Liu, W. He, and Y. Li, "A new digital watermarking algorithm based on WBCT," Procedia Engineering, vol. 29, pp. 1559-1564, 2012.

[11] F. Rahimi and H. Rabani, "A visually imperceptible and robust image watermarking scheme in contourlet domain," in Proceedings of the 2010 IEEE 10th International Conference on Signal Processing, ICSP2010, pp. 1817-1820, Beijing, China, 2010.

[12] S.-M. Zhu and J.-M. Liu, "Novel blind watermarking scheme in contourlet domain based on singular value decomposition," in Proceedings of the 2nd International Workshop on Knowledge Discovery and Data Mining (WKKD '09), pp. 672-675, Moscow, Russia, 2009.

[13] G. Easley, D. Labate, and W.-Q. Lim, "Sparse directional image representations using the discrete shearlet transform," Applied and Computational Harmonic Analysis. Time-Frequency and Time-Scale Analysis, Wavelets, Numerical Algorithms, and Applications, vol. 25, no. 1, pp. 25-46, 2008.

[14] P. Kittipoom, G. Kutyniok, and W.-Q. Lim, "Construction of compactly supported shearlet frames," Constructive Approximation. An International Journal for Approximations and Expansions, vol. 35, no. 1, pp. 21-72, 2012.

[15] B. Ahmederahgi, F. Kurugollu, P. Milligan, and A. Bouridane, "Spread spectrum image watermarking based on the discrete shearlet transform," in Proceedings of the 4th European Workshop on Visual Information Processing (EUVIP '13), pp. 178-183, Paris, France, June 2013.

[16] J. Zhao, M. Sun, J. Jia, L. Huang, F. Han, and S. Liu, "Image watermark based on extended shearlet and insertion using the largest information entropy on horizontal cone," Mathematical Problems in Engineering, vol. 2015, Article ID 450819, 10 pages, 2015. 
[17] M. Mardanpour and M. A. Z. Chahooki, "Robust transparent image watermarking with Shearlet transform and bidiagonal singular value decomposition," AEU-International Journal of Electronics and Communications, vol. 70, no. 6, pp. 790-798, 2016.

[18] X. Wang, Y. Liu, S. Li, H. Yang, and P. Niu, "Robust image watermarking approach using polar harmonic transforms based geometric correction," Neurocomputing, vol. 174, pp. 627-642, 2016.

[19] C.-C. Lin, C.-C. Chang, and Y.-H. Chen, "A novel SVD-based watermarking scheme for protecting rightful ownership of digital images," Journal of Information Hiding and Multimedia Signal Processing, vol. 5, no. 2, pp. 124-143, 2014.

[20] R. Mehta and N. Rajpal, "A hybrid semi-blind gray scale image watermarking algorithm based on DWT-SVD using human visual system model," in Proceedings of the 6th International Conference on Contemporary Computing, (IC3 '13), pp. 163-168, Noida, India, 2013.

[21] B. L. Gunjal and S. N. Mali, "MEO based secured, robust, high capacity and perceptual quality image watermarking in DWTSVD domain," SpringerPlus, vol. 4, no. 1, pp. 1-16, 2015.

[22] A. Benhocine, L. Laouamer, L. Nana, and A. C. Pascu, "New images watermarking scheme based on singular value decomposition," Journal of Information Hiding and Multimedia Signal Processing, vol. 4, no. 1, pp. 9-18, 2013.

[23] K. Loukhaoukha, "On the security of digital watermarking scheme based on SVD and tiny-GA," Journal of Information Hiding and Multimedia Signal Processing, vol. 3, no. 2, pp. 135141, 2012.

[24] M. M. Islam, D. Zhang, and G. Lu, "A geometric method to compute directionality features for texture images," in Proceedings of the IEEE International Conference on Multimedia and Expo, (ICME '08), pp. 1521-1524, Hannover, Germany, 2008.

[25] Y.-W. Kim and I.-S. Oh, "Watermarking text document images using edge direction histograms," Pattern Recognition Letters, vol. 25, no. 11, pp. 1243-1251, 2004. 


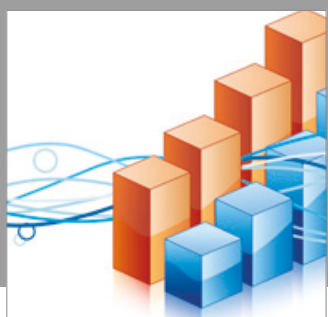

Advances in

Operations Research

vatersals

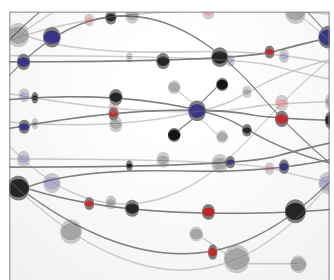

\section{The Scientific} World Journal
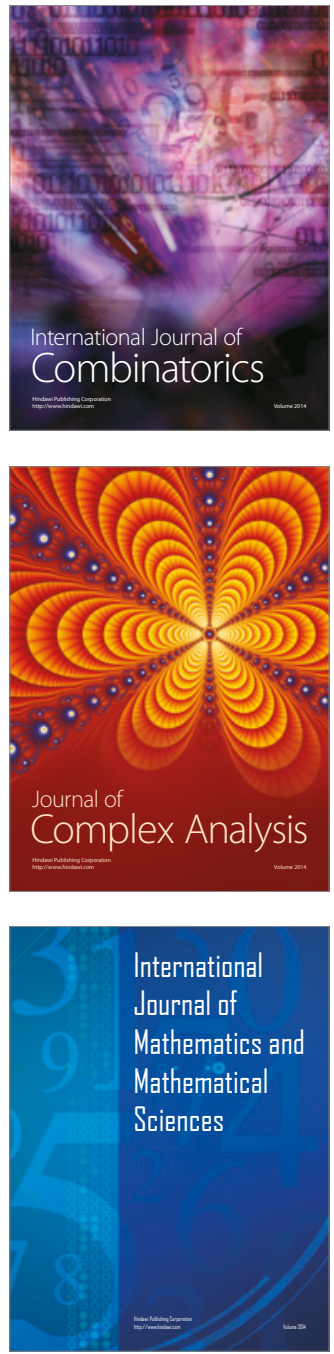
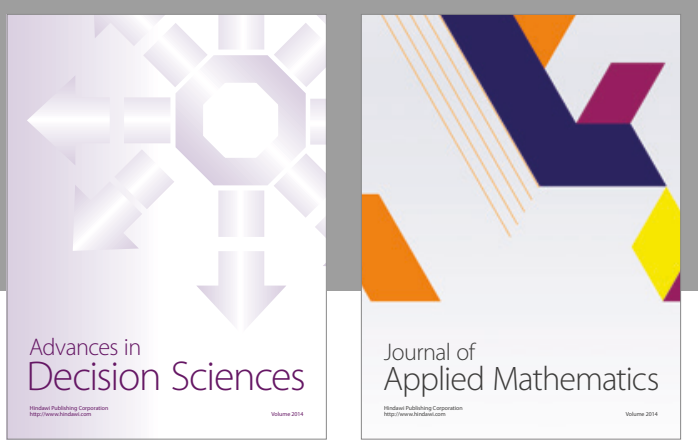

Algebra

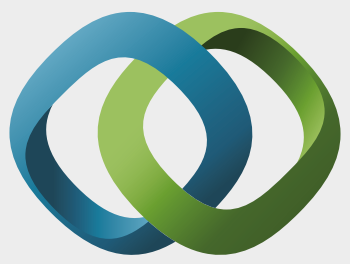

\section{Hindawi}

Submit your manuscripts at

https://www.hindawi.com
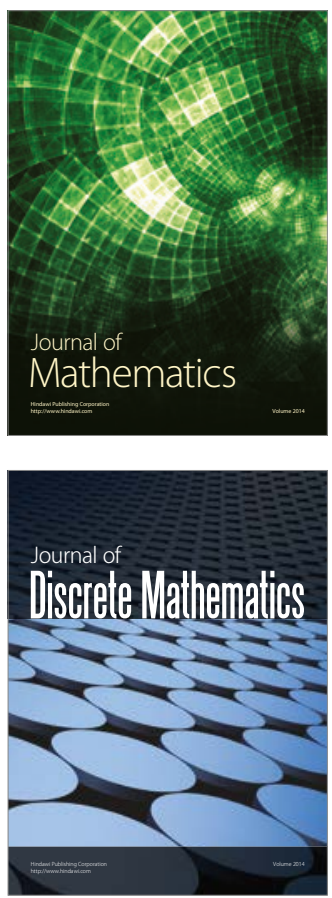

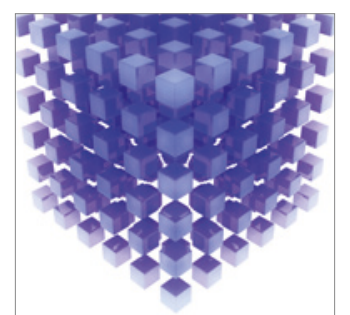

Mathematical Problems in Engineering
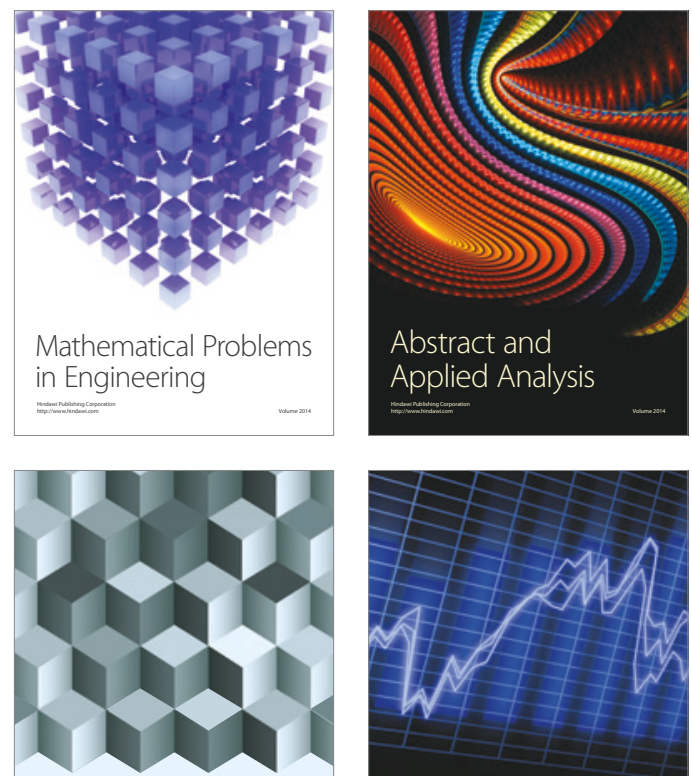

Journal of

Function Spaces

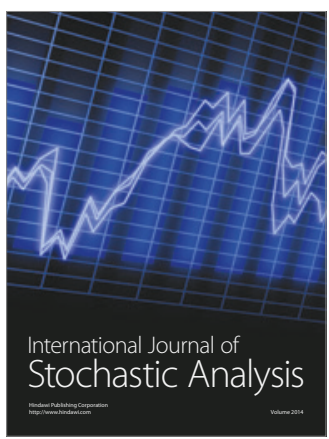

Probability and Statistics
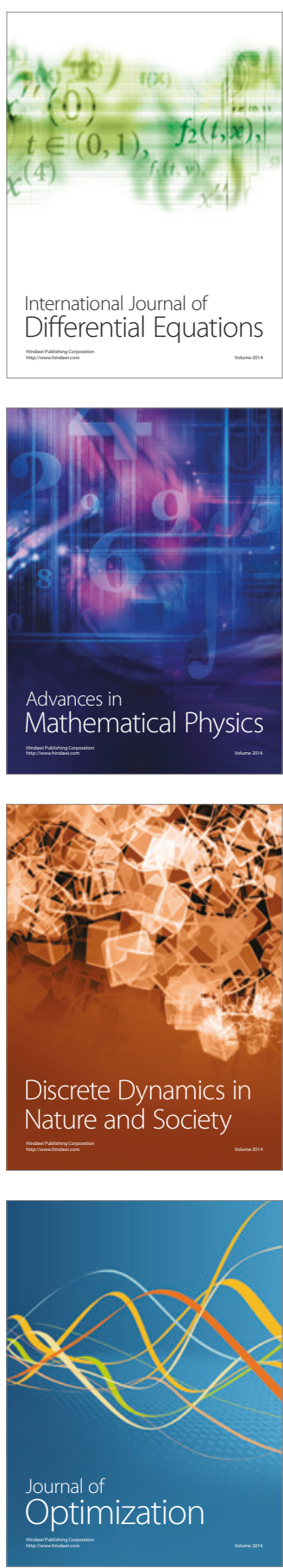